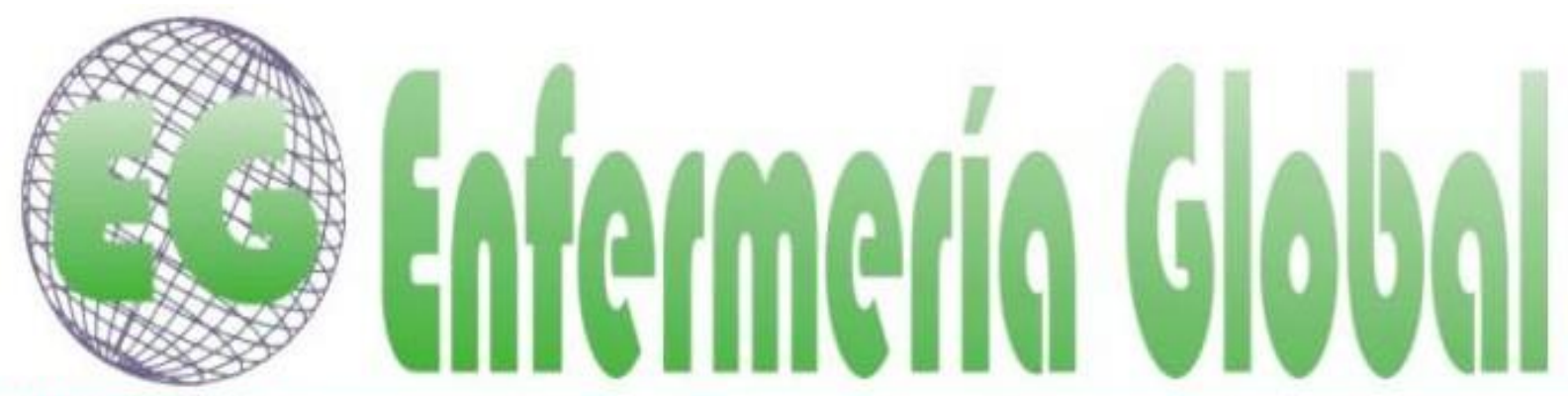

\title{
Edad, Género y Resiliencia en la Conducta Sexual de Riesgo para ITS en Adolescentes al Sur de México
}

Age, Gender and Resilience in Sexual Risk Behavior of STI among adolescents in Southern Mexico

\section{${ }^{*}$ Castillo-Arcos, Lubia del Carmen ${ }^{* *}$ Alvarez-Aguirre, Alicia ${ }^{* * *}$ Bañuelos- Barrera, Yolanda ${ }^{* * * *}$ Valle-Solís, Martha Ofelia ${ }^{* * * * *}$ Valdez-Montero, Carolina *Kantún-Marín, María Amparo de Jesús}

\begin{abstract}
*Doctora en Ciencias de Enfermería. Universidad Autónoma del Carmen, Facultad Ciencias de la Salud. E-mail lubiacastilloa@gmail.com **Doctora en Ciencias de Enfermería. Universidad Autónoma de Querétaro. ***Doctora en Ciencias de Enfermería. Universidad Juárez del Estado de Durango. ***Doctora en Ciencias de Enfermería. Universidad Autónoma de Nayarit. **** Doctora en Ciencias de Enfermería. Universidad Autónoma de Sinaloa. México.
\end{abstract}

http://dx.doi.org/10.6018/eglobal.16.1.234921

\section{RESUMEN}

Objetivo: Comparar las conductas sexuales de riesgo en estudiantes de preparatoria y diferenciar por edad, género y nivel de resiliencia sexual.

Material y métodos: Estudio descriptivo, transversal y explicativo. La población de interés estuvo conformada por 182 adolescentes de 15 y 16 años de dos instituciones educativas. La selección de la muestra fue mediante un muestreo aleatorio estratificado.

Resultados: Los instrumentos de medición mostraron medidas de confiabilidad aceptables. El $69 \%$ de los adolescentes refirió contar con 16 años de edad, mientras que el 64\% correspondió al género femenino. Los adolescentes de 16 años tuvieron promedios más altos en las conductas sexuales de riesgo $(M=12.1, D E=23.3)$ que los adolescentes de 15 años $(M=4.76, D E=14.6, U=2,984.0$, $p=.038)$. En cuanto al género se presentaron diferencias significativas, las mujeres presentaron $(M=$ 7.45, $D E=2.77)$ los hombres $(M=8.01, D E=2.03, U=3,714.0, p=.017)$. Mientras que el nivel de resiliencia sexual también fue diferente, los adolescentes de 15 años mostraron niveles de resiliencia más bajos $(M=60.5, D E=13.6)$ que los adolescentes de 16 años $(M=65.0, D E=17.3 ; U=2,809.0$, $p=.034$ ). El Modelo de Regresión Lineal identificó que las variables que mayor contribución tuvieron fueron la edad, género y estado civil para la conducta sexual de riesgo $\left(F=1.85, R^{2}\right.$ ajustada $=.065$, $p=.052$ ). En otro modelo la edad, genero y estado civil fueron significativas para el desarrollo de la 
resiliencia $\left(F=5.4, R^{2}\right.$ ajustada $\left.=.07, p=.001\right)$. La investigación sobre la conducta sexual del adolescente y los factores que influyen en el comportamiento, es útil para comprender lo que motiva a los adolescentes en participar o evitar las conductas de riesgo sexual.

Palabras clave: Palabras clave: Resiliencia; Conducta sexual de riesgo; ITS (Fuente: DeCs, Bireme)

\section{ABSTRACT}

Objective: To compare the sexual risk behavior in high school students and differentiate by age, gender and level of sexual resilience.

Methods: Descriptive, transversal and explanatory study. The target population consisted of 182 adolescents aged 15 to 16 years of two educational institutions. The selection of the sample was using a stratified random sampling.

Results: Measuring instruments showed acceptable reliability measures. $69 \%$ of adolescents reported having 16 years of age, while $64 \%$ were female. Teenagers of 16 years had higher averages in sexual risk behaviors $(M=12.1, S D=23.3)$ than adolescents of 15 years $(M=4.76, S D=14.6, U=2984.0, p$ $=.038)$. Regarding gender, significant differences were found, women had $(M=7.45, S D=2.77)$ for men $(M=8.01, S D=2.03, U=3714.0, p=.017)$. While the level of sexual resilience was also different, teens 15 years showed lower levels of resilience $(M=60.5, S D=13.6)$ than adolescents of 16 years $(M$ $=65.0, S D=17.3 ; U=2809.0, p=.034)$. The Linear Regression Model identified that the greatest contribution were variables were age, gender and marital status to sexual risk behavior $(F=1.85$, $R^{2}$ ajustada $\left.=.065, p=.052\right)$. In another model age, gender and marital status were significant for the development of resilience $\left(F=5.4, R^{2}\right.$ ajustada $\left.=.07, p=.001\right)$. Research on adolescent sexual behavior and the factors that influence behavior, it is useful to understand what motivates adolescents to participate or avoid sexual risk behaviors.

Keywords: resilience; sexual risk behavior; ITS (Source: DeCs, Bireme)

\section{INTRODUCCIÓN}

Las Infecciones de Transmisión Sexual (ITS) afecta a diversos grupos vulnerables de manera preocupante y a pesar de las campañas emprendidas para disminuir los casos de ITS, entre ellas el Virus de la Inmunodeficiencia Humana $(\mathrm{VIH})$, se siguen manteniendo. A nivel mundial la epidemia del VIH se ha mantenido estable; sin embargo, los nuevos casos y las muertes por esta infección continúan en algunos países de manera alarmante. Datos del Programa Conjunto de las Naciones Unidas sobre el $\mathrm{VIH}^{1}$ indica que en el 2013 existían 35 millones de personas con $\mathrm{VIH}, 2.1$ millones contrajeron la enfermedad y 1.5 millones murió por esta causa. Asimismo, el organismo menciona que en caso de no disminuir el VIH en los próximos cinco años, la epidemia resurgirá con una incidencia mayor a la que existe actualmente. El $45 \%$ de las personas que viven con el VIH se concentran en América Latina ${ }^{2}$, de ellos un porcentaje importante es representado por adolescentes y jóvenes (45\%).

Por otra parte, los adolescentes con frecuencia tienen pensamientos erróneos sobre sexualidad y consideran que tener relaciones sexuales no es de riesgo, son impulsivos, tienden a fantasear; por lo que no consideran las consecuencias de sus acciones y ante los cambios en su salud no buscan la atención médica oportuna. Por lo anterior, la salud sexual del adolescente es un tema de interés en los sistemas de salud de los países de ingreso medio y bajo, debido a que las ITS van en aumento a pesar de las campañas de prevención implementadas de manera masiva ${ }^{3,4}$. Diversos autores ${ }^{5,6,7}$ consideran que los adolescentes que inician las relaciones sexuales a temprana edad y los que piensan que son demasiado jóvenes para enfermar y morir, tienen mayor predisposición para adquirir alguna ITS, por lo que se vuelve un grupo vulnerable. 
En este sentido, existen diversos factores que pueden predisponer al riesgo de ITS en la adolescencia, uno de ellos es el género, el cual es considerado como la vivencia de los significados relacionados a la sexualidad; las construcciones de los conceptos y los roles que debe asumir el hombre y la mujer están enmarcados por las reglas sociales y culturales. Lo anterior, sustenta el patrón sexual específico y el poder que ejerce determinado género en las relaciones de pareja ${ }^{8}$. También, la edad es un factor determinante en la práctica de conductas de riesgo, diversos autores refieren que el inicio de relaciones sexuales a menor edad, se convierte en un mayor riesgo de embarazos e ITS, ya que el adolescente aún se encuentra en una evolución cognitiva de construcción y reconstrucción de sus pensamientos, ideas y personalidad que regularán el comportamiento sexual ${ }^{5,9}$.

Por otro lado, la resiliencia sexual favorece la conducta segura, bajo la influencia positiva de esta el adolescente ejerce comportamientos responsables y autodeterminados, al desarrollar la capacidad de responder de manera crítica y resolutiva ante diversas situaciones de riesgo sexual e influencias nocivas. A su vez, estimula en el adolescente la necesidad de mejorar sus condiciones de salud. El desarrollo de la capacidad resiliente en los adolescentes se convierte en una oportunidad única para el personal de salud involucrado en la atención sexual de este grupo de población, ya que permite modular el efecto del riesgo ${ }^{10}$. Por lo anterior, se planteó el objetivo comparar las conductas sexuales de riesgo en estudiantes de preparatoria y las diferencias por edad, género y nivel de resiliencia sexual.

\section{METODOLOGÍA}

Estudio descriptivo, transversal y explicativo ${ }^{11}$. La población de interés estuvo conformada por 182 adolescentes de 15 y 16 años de dos instituciones educativas del estado de Campeche, México. La selección de la muestra fue mediante un muestreo aleatorio estratificado, con un poder estadístico de $90 \%$.

\section{Selección de participantes}

Criterios de inclusión: adolescente que aceptaran participar voluntariamente en el estudio y con el consentimiento de sus padres. Criterios de exclusión: adolescentes casados o que vivieran en unión libre. Criterios de eliminación: cuestionarios incompletos.

\section{Instrumentos}

Se aplicó una cédula de datos sociodemográficos para describir a los participantes del estudio. También, se utilizaron dos instrumentos para medir los conceptos de interés. El primero fue la Escala de Conducta Sexual ${ }^{12,13}$, mide la frecuencia del uso de condón, de tener sexo con diferentes parejas y conducta sexual en los pasados 3 meses, está integrada de 38 reactivos, con un rango de puntuación total de 0 a 76 . El segundo fue la escala de resiliencia sexual ${ }^{14,15}$, la escala mide el nivel de resiliencia para sexo seguro, está formada por 22 ítems, con rango de $22-110$.

\section{Procedimiento}

El protocolo fue presentado ante el Comité de Investigación de la Facultad de Enfermería de la Universidad Autónoma del Carmen. Se obtuvo la autorización del personal directivo de las Instituciones Educativas para la realización del estudio, 
posteriormente se dio información a los padres de familia sobre el proyecto en ambas instituciones, obteniéndose el consentimiento de cada uno de ellos y el asentimiento informado de los adolescentes. A continuación se programó con las autoridades de la escuela la recolección de los datos, los cuales fueron recolectados por pasantes en servicio social de la licenciatura en enfermería previamente adiestrados para este fin. A los adolescentes se les informó del carácter anónimo y voluntario del cuestionario. Se leyeron las indicaciones en voz alta, aclarando las dudas que se presentaron, se realizó hincapié en que no dejaran preguntas sin contestar. Durante la aplicación de los cuestionarios los encuestadores estuvieron presentes. Al finalizar los adolescentes colocaron los cuestionarios en una urna para asegurar la confidencialidad y anonimato de la información.

\section{Análisis estadístico}

Los datos estadísticos se analizaron mediante el Statistical Package for the Social Sciences (SPSS), versión 17 para Windows, se aplicó estadística descriptiva e inferencial, para las variables categóricas se calculó frecuencias y porcentajes y en las variables cuantitativas medidas de tendencia central y dispersión. Posterior a la verificación de la distribución de las variables, se usó el estadístico de prueba $U$ de Mann Whitney para verificar diferencia entre ellas Se realizaron Modelos de Regresión Lineal. Asimismo, para demostrar significancia estadística de los resultados el valor de $p$ fue $<0.05$.

\section{Aspectos éticos}

Para la realización del estudio se tomó en cuenta lo establecido en el Capítulo I, artículo 13 del Reglamento de la Ley General de Salud en Materia de Investigación para la Salud ${ }^{16}$, de los aspectos éticos de la investigación en seres humanos. Se respetó la dignidad, anonimato, protección de los derechos y bienestar de los participantes en el estudio, durante el reclutamiento, selección de los participantes y recolección de los datos. La participación de los adolescentes solo fue del conocimiento del investigador principal, los colaboradores del proyecto, los padres y los otros, así mismo la información colectada solo fue manejada por el investigador principal y se dio a conocer en forma general.

\section{RESULTADOS}

Los datos corresponden a 182 adolescentes de nivel preparatoria, de los cuales, el $69 \%$ refirió contar con 16 años de edad, mientras que el 64\% correspondió al género femenino. De los participantes, el $90 \%$ cursaba el segundo semestre y el $68 \%$ mencionaron ser solteros. Respecto al número de personas de la familia que vivían en el mismo domicilio, el $67 \%$ informó que vivían entre 4 y 5 personas por domicilio, de las cuales se incluyó al padre y a la madre (75\% y $99 \%$ respectivamente) y un hermano ó hermana en el $60 \%$ y $55 \%$ de los casos (ver tabla 1 ). 
Tabla 1 Características sociodemográficas

\begin{tabular}{|c|c|c|}
\hline Variable & $f$ & $\%$ \\
\hline \multicolumn{3}{|l|}{ Sexo } \\
\hline Femenino & 166 & 63.7 \\
\hline Masculino & 64 & 35.2 \\
\hline \multicolumn{3}{|l|}{ Edad } \\
\hline 15 & 56 & 30.8 \\
\hline 16 & 126 & 69.2 \\
\hline \multicolumn{3}{|l|}{ Semestre } \\
\hline $1 \stackrel{0}{0}$ & 17 & 9.3 \\
\hline $2^{\circ}$. & 163 & 89.6 \\
\hline \multicolumn{3}{|l|}{ Estado Civil } \\
\hline Soltero & 124 & 68.1 \\
\hline $\begin{array}{l}\text { En relación } \\
\text { de noviazgo }\end{array}$ & 56 & 30.8 \\
\hline \multicolumn{3}{|l|}{ Experiencia Sexual } \\
\hline No & 153 & 84.1 \\
\hline $\mathrm{Si}$ & 27 & 14.8 \\
\hline No respondieron & 2 & 1.1 \\
\hline
\end{tabular}

Nota: $f$ = Frecuencia; $\%$ = Porcentaje

Al cuestionarles si habían recibido información sobre sexualidad en los últimos tres meses, el $87 \%$ respondieron afirmativamente, a la vez que el $91 \%$ recibió específicamente información sobre las ITS. Por otra parte, el $83.5 \%$ reportó haber recibido información sobre VIH o Sida, mientras que el $98 \%$ nunca se había realizado la prueba rápida para detectar $\mathrm{VIH}$.

Se obtuvo la confiabilidad de las escalas con el alfa de Cronbach y presentaron alfas de .97 y .87 para las escalas de conductas sexuales de riesgo y la resiliencia sexual, las cuales se consideran aceptables. Con respecto a las escalas utilizadas, el nivel de conductas sexuales de riesgo mostró un promedio de $9.8(D E=21.3)$ y la escala de resiliencia sexual mostró un promedio de $63.6(D E=16.3)$

El objetivo planteó comparar las conductas sexuales de riesgo en estudiantes de preparatoria y diferenciar por edad, género y nivel de resiliencia sexual. Para corroborarlo, se utilizó el estadístico de prueba $U$ de Mann Whitney, cuyos resultados mostraron para la edad, que los adolescentes de 16 años tuvieron promedios más altos en las conductas sexuales de riesgo $(M=12.1, D E=23.3)$ que los adolescentes de 15 años $(M=4.76, D E=14.6)$ cuya diferencia resultó estadísticamente significativa $(U=2,984.0, p=.038)$. En cuanto al género los puntajes presentaron una variación mínima las mujeres presentaron $(M=7.45, D E=2.77)$ y los hombres $(M=$ $8.01, D E=2.03)$ cuya diferencia resultó estadísticamente significativa $(U=3,714.0, p$ $=.017)$

Mientras que el nivel de resiliencia sexual también fue diferente, los adolescentes de 15 años mostraron niveles de resiliencia más bajos $(M=60.5, D E=13.6)$ que los adolescentes de 16 años $(M=65.0, D E=17.3)$ que también fue estadísticamente significativo $(U=2,809.0, p=.034)$ (ver tabla 2). Al comparar por género, se encontró que los hombres mostraron niveles más altos $(M=69.5, D E=13.3)$ de resiliencia sexual que las mujeres $(M=60.2, D E=17.1)$ cuya diferencia fue estadísticamente significativa $(U=2,532.5, p=.001$; ver tabla 2$)$. 
Tabla 2 Prueba $U$ de Mann-Withney para conducta sexual de riesgo y resiliencia sexual

\begin{tabular}{lcccc}
\hline Variable & Media & $D E$ & $U$ & $p$ \\
\hline Conducta Sexual de riesgo & & & $2,984.0$ & .038 \\
$\quad 15$ años & 4.76 & 14.6 & & \\
$\quad 16$ años & 12.14 & 23.3 & & \\
$\begin{array}{l}\text { Conducta Sexual de riesgo } \\
\quad \text { Femenino }\end{array}$ & 7.45 & 2.77 & & \\
$\quad$ Masculino & 8.01 & 2.03 & & \\
Resiliencia Sexual & & & $2,809.0$ & .034 \\
$\quad 15$ años & 60.57 & 13.6 & & \\
$\quad 16$ años & 65.05 & 17.3 & & \\
Resiliencia Sexual & & & $2,532.5$ & .001 \\
$\quad$ Femenino & 60.25 & 17.1 & & \\
$\quad$ Masculino & 69.54 & 13.3 & & \\
$\begin{array}{l}\text { Nota: Media = Promedio aritmético; DE = Desviación estándar; } U=U \text { de Mann-Withney; } p=\text { Valor de } \\
\text { significancia }\end{array}$
\end{tabular}

En un análisis adicional, se ajustó un Modelo de Regresión Lineal (MRL) en el que se introdujo como variables independientes $(\mathrm{VI})$ las características sociodemográficas y el antecedente de sexualidad, Conocimiento sobre ITS, VIH y Sida, y como variable dependiente (VD) las conductas sexuales de riesgo, el cual tendió a la significancia ( $F$ $=1.85, R^{2}$ ajustada $=.065, p=.052$ ). Al analizarlo, se identificó que las variables que mayor contribución fueron la edad, género y estado civil, por lo que se ajustó otro MRL donde se incluyeron esas variables como VI y la conducta sexual de riesgo como VD. El modelo resultó significativo al encontrar que el $8.4 \%$ de la variación en las conductas sexuales de riesgo se debió a la edad, género y estado civil $(F=6.38$, $R^{2}$ ajustada $=.084, p=.000$ ).

Por otra parte, en otro MRL se introdujeron la edad, género y estado civil como VI y el nivel de resiliencia sexual como VD, el cual resultó significativo $\left(F=5.4, R^{2}\right.$ ajustada $=$ $.07, p=.001$ ), al encontrar que el $7 \%$ de la variación en el nivel de resiliencia sexual, se debió a la influencia de las variables independientes del modelo.

\section{DISCUSIÓN}

Las conductas sexuales en los adolescentes es un tema que cada día se vuelve más relevante por las implicaciones que conlleva para la salud de este grupo de población. De acuerdo a diversos estudios y a los datos estadísticos alarmantes sobre ITS-VIH, embarazos no deseados, abandono escolar, exclusión social, entre otros, el problema sexual se agrava y al ser de origen conductual los riesgos siempre estarán latentes para el adolescente. Por tal motivo, se realizó la presente investigación para conocer las conductas sexuales de riesgo en estudiantes de preparatoria y las diferencias por edad, género y nivel de resiliencia sexual.

De acuerdo a los resultados encontrados se observa que las mujeres fueron más participativas, esto posiblemente se deba a la cultura latina donde la mujer muestra mayor participación para cuidar su salud, lo anterior coincide con otros estudios ${ }^{17,18}$.

Por otro lado, un factor protector para prevenir las conductas sexuales de riesgo es el apoyo de la familia. En el presente estudio se encontró que la mayoría de los 
adolescentes viven con los padres, en ella los adolescentes son apoyados económica y emocionalmente y esta representa el primer pilar de apoyo. Los integrantes de la familia fortalecen entre ellos la protección, comunicación, amor y cariño, esto constituye un vínculo muy fuerte que hace que el adolescente ante situaciones de riesgo pueda acudir a su familia en busca de apoyo y orientación; lo anterior, coincide con diversos autores ${ }^{19,20}$ quienes refieren que los adolescentes perciben a la familia como primera fuente de apoyo, cuando presentan dudas recurren a ella para obtener información. Asimismo, este apoyo es más percibido por los adolescentes que no tienen una vida sexual activa.

También, se encontró que los adolescentes en su mayoría han recibido información sobre sexualidad, ITS-VIH y Sida en los últimos tres meses, lo que representa otro factor protector vinculado al conocimiento, que le permite en esta etapa de madurez cognitiva interiorizar datos relevantes que lo ayudarán a tomar conciencia de su salud sexual y los riesgos a los cuales se verá inmerso debido al momento histórico en el que vive. Sin embargo, un bajo porcentaje de adolescentes reportaron no tener información sobre estos temas, situándolos en una posición de riesgo. Lo que es consistente con otros autores 19,21 quienes mencionan que en la etapa de la adolescencia hay una necesidad de recibir mayor información sobre temas de sexualidad, para lograr estrategias de autocuidado. Sin embargo, tener información no equivale a tener conocimiento correcto para prevenir las ITS.

A su vez, se encontró que los adolescentes de mayor edad presentaron más probabilidades de practicar conductas sexuales de riesgo; lo anterior, podría deberse a que en esta etapa los adolescentes están en un proceso de cambios constantes, que los hacen vulnerables, con las consecuencias negativas para su salud, lo que concuerda con otros autores ${ }^{18,20}$ quienes refieren que los adolescentes mayores inician su primera relación sexual alrededor de los 15 años. Lo anterior sugiere, que tienen mayor probabilidad de practicar conductas sexuales de riesgo debido a que se encuentran en una etapa de experimentación y descubrimiento de su sexualidad, donde lo prohibido y misterioso se vuelve un reto constante.

En este estudio, el género mostró diferencias significativas en relación a las conductas sexuales de riesgo; los hombres presentaron mayor puntaje. Lo que concuerda con otros estudios ${ }^{8,23}$, donde se encontró que los hombres presentan más conductas de riesgo al iniciar las prácticas sexuales a más temprana edad (15.2 años). Asimismo, los adolescentes hombres son más desinhibidos y debido a las prácticas culturales como la poligamia, parejas ocasionales, el uso del sexo comercial, menos vigilancia de los padres por ser varones, entre otras; tienen una predisposición a conductas de riesgo ${ }^{17}, 24$.

En relación a la resiliencia los adolescentes de mayor edad presentaron mayor nivel de resiliencia. Lo que coincide con otros autores ${ }^{25}$, mencionan que un nivel alto de resiliencia favorece la prevención de conductas sexuales de riesgo. Los resultados mostraron que los hombres son más resilientes que las mujeres, lo que podría indicar que el sexo femenino es más propenso a situaciones de riesgo, lo que concuerda con Matta $^{26}$ en su estudio halló que el sexo masculino posee un grado de resiliencia alto, lo que indica que los hombres tienen mayor capacidad para contrarrestar los factores de riesgo sexual.

Asimismo, se encontró que la edad, género y estado civil son determinantes que contribuyen a las conductas sexuales de riesgo. Diversos estudios han demostrado 
que tener una relación de noviazgo está asociado con el inicio de relaciones sexuales, así como tener mayor edad y ser hombre ${ }^{27,28}$. La influencia de una persona romántica, la edad y el género impacta en la iniciación sexual y la salud sexual del adolescente.

En este sentido, se demostró que la edad, género y estado civil influyen en el desarrollo de la resiliencia sexual, esta última considerada como la capacidad para contrarrestar las situaciones de riesgo a través del uso de los factores protectores que posee la persona, lo que concuerda con Esparza ${ }^{29}$ quien indica que si los adolescente tienen un adecuado nivel de resiliencia pueden tomar decisiones asertivas sobre los cambios que se vayan presentando en cuanto a conductas sexuales. Sin embargo, se encontró en el presente estudio que los adolescentes hombres de mayor edad, con una relación de noviazgo y nivel bajo de resiliencia tiene más probabilidades de practicar conductas sexuales de riesgo.

\section{CONCLUSIONES}

De acuerdo a lo anterior, se puede concluir que los adolescentes tienen más probabilidades de realizar comportamientos de riesgo sexual, debido al proceso vital que cursan y a las normas sociales y culturales que los rigen. El género y la edad juegan un papel importante para direccionar los programas educativos, ya que indica las pautas para intervenir al hombre y la mujer; lo anterior, resulta relevante para el diseño de programas de prevención, que deberán buscar el reforzamiento de la información, mediante diversas estrategias de enseñanza que resulten interesantes y novedosas a los adolescentes; además, generen interés y aprendizaje significativo para la prevención de conductas sexuales de riesgo.

Para el profesional de salud esto representa una oportunidad para dirigir objetivos que ayuden a mejorar la habilidad en la toma de decisiones asertivas para el aplazamiento de la primera relación sexual y la reducción del riesgo en este grupo de población. El profesional de enfermería debe participar en la atención de la salud sexual, para proporcionar atención integral al adolescente, con un enfoque de detección y prevención de los posibles riesgos para la salud física y psicosocial relacionada a comportamientos negativos. Asimismo, las Instituciones de Salud deben habilitar prácticas que favorezcan el acceso libre y confidencial a los servicios de salud sexual.

La investigación sobre la conducta sexual del adolescente y los factores que influyen en el comportamiento, es útil para comprender que motiva a los adolescentes en participar o evitar las conductas de riesgo sexual. Muchos adolescentes están involucrados en una gran cantidad de riesgos; los conocimientos, habilidades y valores que desarrollen en el transcurso de su vida los colocan en la posibilidad de llevar una vida segura y feliz, a diferencia de quien no posea esos elementos tiene más posibilidades de contraer alguna ITS como el VIH; por lo que, aumenta en forma considerable el riesgo de morir a temprana edad. Es por ello, la importancia de ayudar al adolescente a convertirse en un adulto sexualmente saludable, con la capacidad para evitar los riesgos a través de una correcta toma de decisiones.

\section{Agradecimiento:}

Consejo Nacional de Ciencia y Tecnología (CONACyT) [45455]

ORCID

Lubia del Carmen Castillo-Arcos http://orcid.org/0000-0002-4368-4735 


\section{REFERENCIAS}

1. Programa Conjunto de las Naciones Unidas sobre el $\mathrm{VIH} /$ sida (ONUSIDA). Comunicado de prensa. [Citado el 05 enero 2015]. Disponible en: http://www.unaids.org/es/resources/presscentre/pressreleaseandstatementarchive/201 4/november/20141118 PR WAD2014report

2. Programa Conjunto de las Naciones Unidas sobre el VIH/sida (ONUSIDA). Informe sobre $\mathrm{VIH} / \mathrm{sida}$. [Citado el 10 julio 2015]. Disponible en: http://www.unaids.org/es/resources/presscentre/pressreleaseandstatementarchive/201 4/july/20140716prgapreport

3. Pai $\mathrm{H}$, Lee S. Sexual self-concept as influencing intended sexual health behaviour of young adolescent Taiwanese girls. J Clin Nurs. 2012; 21:1988-97

4. Sychareun V, Thomsen S, Chaleunvong K, Faxelid E. Risk perceptions of STIs/HIV and sexual risk behaviours among sexually experienced adolescents in the Northern part of Lao PDR. BMC Public Health. 2013; 13:1126.

5. Monasterio E, Hwang LY, Shafer MA. Adolescent sexual health. Curr Probl Pediatr Adolesc Health Care. 2007; 37(8):302-25.

6. Bradley-Stevenson,C, Mumford J. Adolescent sexual health. Pediatrics Child Health. 2007; 17(12):474-79.

7. Jones R, Bradley E. Health issues for adolescents. Paediatr Child Health. 2007; 17(11): 433-38.

8. García-Vega E, Menéndez E, Fernández P, Cuesta M. Sexualidad, Anticoncepción y Conducta sexual de riesgo en adolescentes. Int J Psychol Res. 2012; 5(1): 79-87.

9. García-Vega E, Menéndez E, Fernández P, Rico F. Influencia del sexo y del género en el comportamiento de una población adolescente 2010; Psicothema, 22(4): 606-12. 10. Díaz G. Consideraciones teóricas acerca del empoderamiento psicológico en salud sexual de actores sexuales vinculados a niños/as preescolares. Rev Cubana Med Integr. 2011; 27(1):23-32.

11. Burns N, Grove S. The practice of nursing research. Appraisal, synthesis, and generation of evidence 6th ed. 2009. St. Louis: Elsevier Saunders.

12. Jemmot, JB III, Jemmott LS, Fong GT. Reduction in HIV risk-associated sexual behaviors among black male adolescents: Effects of an AIDS prevention intervention. Am J Public Health.1992; 82(3): 372-77.

13. Villarruel A M, Jemmontt J B III, Jemmontt L S, Ronis D L. Predictors of sexual intercourse intentions and condom use among Spanish dominant youth: $A$ test of the theory of planned behavior. Nurs Res.2004; 53(3): 172-81.

14. Wagnild GM, Young HM. Development and psychometric evaluation of the resilience scale. J Nurs Meas. 1993; 1(2): 165-78.

15. Castillo-Arcos L, Benavides-TorresR, López-Rosales F. Vol. XIV de la Psicología Social en México. Validación de la escala de resiliencia para sexo seguro, 2012. México.

16. Secretaria de Salud. Reglamento de la ley general de salud en materia de investigación para la salud. 1987. (México). [Citado el 05 enero 2015]. Disponible en: http://www.salud.gob.mx/unidades/cdi/nom/compi/rlgsmis.html

17. Morales-Mesa S, Arboleda-Álvarez O, Segura-Cardona A. Las prácticas sexuales de riesgo en poblaciones universitarias. Rev Salud Publica. 2014; 16(1): 27-39.

18. Fennie T, Laas, A. HIV/AIDS-related Konowledge, Attitudes and Risky Sexual Behavior among a Sample of South African University Students. Gender and Behaviour. 2014; 12(1):6035-44. 
19. Orcasita L, Uribe A, Castellanos L, Gutiérrez M. Apoyo social y conductas sexuales de riesgo en adolescentes de Lebrija-Santander. Rev Psicol. 2012; 30(2): 371-406.

20. Wil E, Koo H. Mother, fathers, son, and daughters: gender differences in factors associated with parent-child communication about sexual topics. Reprod Health 2010; 7:31 [Citado el 30 de septiembre 2015]. Disponible en: http://www.ncbi.nlm.nih.gov/pmc/articles/PMC3019147/

21. Uribe F, Orcasita LT, Vergara T. Factores de riesgo para la infección por VIH/SIDA en adolescents y jovenes colombianos. Act Colom Psicol. (2010); 13(1):11-24.

22. Shishana O, Rehle T, Simbayi LC, Zuma K, Jooste S, Pillay-van-Wyk V, Mbelle N, Van Zyl J, Parker W, Zungu NP, Pezi S \& the SABSSAM III. South African National HIV prevalence, incidence, behavior and communication survey, 2008: A turning tide among teenagers?. Cape Tawn. Human Sciences Research Council. South Africa. 2009. [Citado el 20 julio 2015]. Disponible en: http://www.health-e.org.za/wpcontent/uploads/2013/05/2966e129fc39e07486250fd47fcc266e.pdf

23. Romero-Estudillo E, Gonzáles-Jiménez, E, Mesa-Franco M, García-García I. Gender-based differences in the high-risk sexual behaviours of young people aged 1529 in Melilla (Spain): a cross-sectional study. BMC Public Health; 14:745.

24. Adejumo AO. Influence of psycho-Demographic factors and effectiveness of psycho-behavioral interventions on sexual risk behavior of in-school adolescents in Ibadan, Nigeria. Ife PsychologIA. 2012; 20(1):1-18.

25. Oppong K, Mayer-Weitz A, Petersen I. Correlates of psychological functioning of homeless youth in Accra, Ghana: a cross-sectional study. Int J Ment Health Syst 2015; 9:1.

26. Matta S. Grado de desarrollo de la resiliencia y su relación con los factores protectores y de riesgo, en adolescentes de instituciones educativas de Lima, Ica, Ayacucho Huánuco. Tesis de Maestria. 2010. [Citado el 10 febrero 2015]. Disponible en:

http://cybertesis.unmsm.edu.pelbitstream/cybertesis/3276/1/matta sh.pdf

27. Ott M, Millstein SG, Ofner S, Halpem-Felsher,BL. Greater expectations: adolescent positive motivations for sex. Perspect Sex Reprod Heald. 2006; 38(2): 849.

28. Widdice LE, Cornell JL, Liang W, Halpem-Felsher BL. Having sex and condon use: potencial risk and benefits report by young, sexually inexperienced adolescents. $J$ Adolesc Health. 2006; 39(4): 588-95.

29. Esparza P. Apoyo social percibido para sexo seguro y resiliencia sexual en el adolescente. Tesis de Maestría. 2014. [Citado el 10 abril 2015]. Disponible en: file://C:/Users/HP/Downloads/TESIS\%202014\%20PALOMA.pdf

Recibido: 1 de agosto 2015;

Aceptado: 16 de octubre 2015

ISSN 1695-6141

() COPYRIGHT Servicio de Publicaciones - Universidad de Murcia 